\title{
Atrial adaptive rate pacing in sick sinus syndrome: effects on exercise capacity and arrhythmias
}

Guy A Haywood, Demosthenes Katritsis, Joan Ward, Michelle Leigh-Jones, David E Ward, A John Camm

\begin{abstract}
Objective-To test the hypotheses that adaptive rate atrial (AAIR) pacing: significantly increases maximal exercise capacity, and results in significant suppression of supraventricular and venticular arrhythmia compared with fixed rate atrial (AAI) pacing.

Design-Prospective, randomised, single blind, crossover study with maximal treadmill exercise testing and 24 hour ambulatory electrocardiographic monitoring in AAIR and AAI modes.

Setting-Regional pacing centre.

Patients-30 consecutive patients (mean SD age 65 (12) years) with sick sinus syndrome who required permanent pacing, without evidence of conduction disturbance on 12 lead electrocardiograms or 24 hour ambulatory electrocardiographic monitoring and without other cardiovascular or systemic disease.
\end{abstract}

Interventions-Activity sensing or minute ventilation driven systems (AAI/AAIR) were implanted alternately.

Results-The mean (SD) peak heart rate in AAI mode was 122(28) $v 130(22)$ in AAIR mode $(p<0.02)$ for the whole group and $104(17) v 120(5)(p<0.003)$ for the patients with chronotropic incompetence. Exercise time was $12 \cdot 3(4 \cdot 1) \mathrm{min}-$ utes in AAI and $12.3(3.8)$ minutes in AAIR mode (NS) for the whole group, and $11.4(4.4)$ in AAI and 11.4 (4.0) in AAIR mode (NS) in the chronotropically incompetent patients. There were no significant differences in the Borg scores at peak exercise in AAI $v$ AAIR mode in either group. The frequency per hour of atrial and ventricular arrhythmias showed no significant differences between the two modes in either the group as a whole or in the subgroups with chronotropic incompetence.

Conclusion-AAIR pacing confers little benefit in sick sinus syndrome compared with AAI pacing.

(Br Heart $\mathcal{f} 1993 ; 69: 174-178)$

Atrial pacing has been proposed as the optimal mode for the management of patients with sick sinus syndrome in the absence of actual or threatened atrioventricular block $\mathrm{k}^{1-3}$ and the implantation of atrial adaptive rate (AAIR) systems has been recommended as the most appropriate mode in patients with sick sinus syndrome and chronotropic incompetence by both the American Heart Association and the American College of Cardiology Task Force on pacemaker implantation $^{4}$ and the British Pacing and Electrophysiology Group Working Party. ${ }^{5}$ These recommendations are questionable, however, as there has been no systematic evaluation of AAIR pacing in these patients.

Comparisons of atrial and ventricular pacing in sick sinus syndrome have shown that fixed rate atrial (AAI) pacing results in a lower rate of progression to chronic atrial fibrillation and that thromboembolic events are less frequent. ${ }^{6}$ The principal concern over the use of atrial pacing is, however, the rate of progression to symptomatic atrioventricular block, which remains controversial. ${ }^{6-8}$ As a result some centres favour dual chamber pacing as an alternative solution. ${ }^{9}$

Chronotropic incompetence, the inability of the sinus node to accelerate in response to exercise, is thought to be present in about one third of patients with sick sinus syndrome. ${ }^{10} 11$ It has been proposed that it is appropriate in these patients to attempt to achieve improved exercise tolerance and suppression of atrial arrhythmias by AAIR pacing. ${ }^{4}$ Preliminary reports on small numbers of patients have suggested that AAIR pacing may confer benefits in terms of capacity to exercise,,$^{1213}$ and suppression of arrhythmia, ${ }^{14}$ but there has been no adequate assessment of the impact of AAIR pacing on the functional capacity of the cardiovascular system in terms of duration of maximal exercise or on the suppression of atrial arrhythmias. We sought to obtain such information by means of a randomised, single blind crossover study.

\section{Patients and methods}

Consecutive patients with sick sinus syndrome, defined as documented sinus bradycardia of $<40$ beats/min or multiple sinus pauses of $>2.5 \mathrm{~s}$ in the presence of symptoms such as dizziness or syncope, were assessed for suitability for atrial pacing. Twelve lead electrocardiograms and 24 ambulatory electrocardiographic monitoring tapes were inspected for evidence of bundle branch block, or first, second, or third degree AV block and if these features were present, either permanently or intermittently, patients were excluded. Patients were also excluded if they lived too far from our centre to permit the travelling required for follow up assessment or if they were known to have other car- 
diovascular or systemic diseases. All patients included in the study were in the New York Heart Association functional class I, and none had clinical signs of heart failure or cardiomegaly on chest $x$ ray film.

Thirteen male and 17 female patients, mean (SD) age $65(12)$ years, satisfied the inclusion and exclusion criteria and were admitted for implantation of programmable AAIR systems. ${ }^{15}$ Medtronic Activitrax II and Telectronic Meta MV generators with their recommended atrial leads, were implanted alternately. (There was one exception where, due to availability, a Medtronic Legend generator had to be substituted for an Activitrax II.)

At the assessment before discharge patients were randomly allocated to either AAI mode at 70 beats/min or AAIR mode at 70 to 140 beats per min for patients under 65 years of age and 70 to 130 beats per min for patients over 65. The Activitrax II system was programmed initially to a rate response of 7 with medium activity threshold for those under 65, and low activity threshold for those over 65 . (If this resulted in an inadequate rate response at the first exercise test in AAIR mode, the rate response and activity threshold were increased during the second AAIR period.) The Meta MV system was programmed to a rate response setting of 22 during the first AAIR period and the optimal setting found at the first exercise assessment by using the peak exercise measurement in the adaptive mode setting as recommended in the manufacturer's manual for physicians.

Assessments including 24 hour ambulatory monitoring and exercise testing was carried out at one, two, three and six months after implantation and the pacing mode was crossed over at each assessment (fig). The chronotropic assessment exercise protocol $(\text { CAEP })^{16}$ was used for all exercise tests (appendix 1). At each stage of the exercise protocol, 15 seconds before change to the next stage of exercise, the patient was shown a Borg score chart (appendix 2) and asked to select a number indicating their subjective experience of the severity of exercise. The peak exercise Borg scores from the last two exercise tests were compared. Patients were not aware of which mode they were in at the time of exercise, and were unable to see any clock or display of duration of exercise. A uniform method of encouragement was used

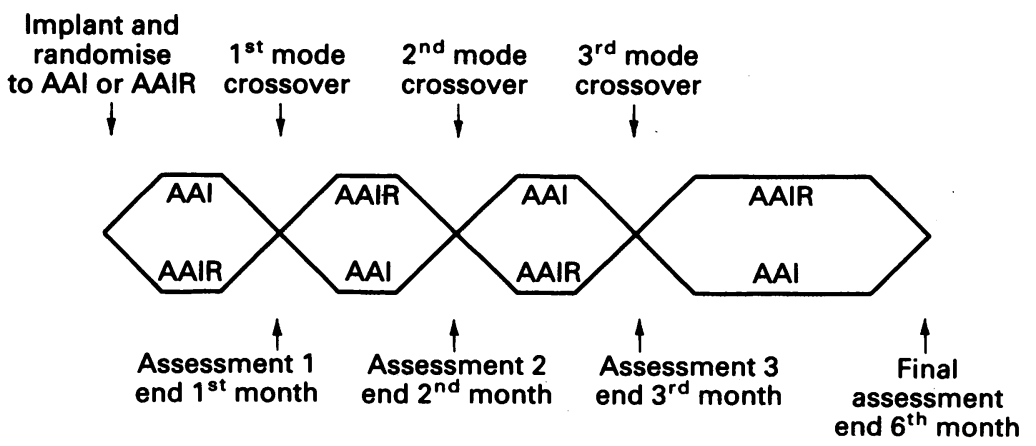

Figure Protocol design. in each test in which the patient was asked if they could continue for a little longer each time they requested to stop; only when the patient considered that they could not carry on longer was the test ended. All tests were supervised by the same personnel.

The fastest heart rate in sinus rhythm achieved on either of the AAI exercise tests was used to determine whether the patient was chronotropically incompetent. The presence of chronotropic incompetence was determined with both currently used definitions-namely, peak heart rate $<0.8$ (220 age of the patient in years) ${ }^{17}$ and peak heart $<$ $0.75(220$ - age in years $){ }^{18}$ These seem to be appropriate definitions across a wide range of ages when used with the CAEP, ${ }^{16}$ and Wilkoff and colleagues found a range of peak heart rates 0.76 to $1.19 \times(220-$ age $)$ in a population of 410 normal subjects with this protocol. ${ }^{16}$ We were unable to examine the prevalence of chronotropic incompetence at submaximal levels of exercise as the formula developed by Wilkoff et $a l^{16}$ requires the resting sinus rate. Most of the patients in our study were paced at rest and some patients with the Meta MV system had resting sinus rates below the lowest rate setting that could be programmed.

Where $P$ waves could be identified clearly among baseline interference at peak exercise, the PR intervals in AAI and AAIR modes were measured at rest and at peak exercise. Ambulatory monitoring tapes were recorded with a Marquette 8500 series Holter monitor and analysed on a Marquette Laser Holter XP. Supraventricular and ventricular arrhythmias were recorded and the frequency per hour of atrial premature beats, couplets, and runs of tachyarrhythmias were calculated. The criterion for defining atrial premature beats was $20 \%$ prematurity compared with the preceding $R R$ interval. All episodes of three or more consecutive premature beats were recorded as runs. Accuracy of analysis was verified manually throughout each recording by one of us (M L-J).

\section{STATISTICAL ANALYSIS}

All paired data were analysed with both the paired $t$ test and the Wilcoxon signed rank test. Comparisons between groups were made with the two tailed Mann-Whitney $U$ test. $p$ values $<0.05$ were considered significant.

\section{Results}

EXERCISE PERFORMANCE

All patients performed at least one exercise test in the AAI mode. Fifteen were chronotropically incompetent when defined by peak heart rate $<0.8(220$ - age in years $)$ and 11 when defined by peak heart rate < 0.75 (220 - age in years). Four patients failed to complete exercise tests and 24 hour ambulatory monitoring tapes in both modes. This was due to revision of the system after progression to symptomatic atrioventricular block in two patients, a telemetry failure in one patient, and refusal to perform repeat 
Table 1 Exercise data (mean (SD)) for whole group and patients with chronotropic incompetence (CI) with peak heart rate in $A A I<80 \%$, and $<75 \%$ of the age predicted maximum value

\begin{tabular}{|c|c|c|c|c|c|c|c|c|c|}
\hline \multirow[b]{2}{*}{ Mode } & \multicolumn{3}{|l|}{ Whole group } & \multicolumn{3}{|c|}{$\begin{array}{l}C I \\
0.8(220-\text { age })\end{array}$} & \multicolumn{3}{|c|}{$\begin{array}{l}C I \\
0.75(220-\text { age })\end{array}$} \\
\hline & $\begin{array}{l}\text { Peak } \\
\text { heart } \\
\text { rate } \\
\text { (beats/min) }\end{array}$ & $\begin{array}{l}\text { Exercise } \\
\text { time } \\
\text { (mins) }\end{array}$ & $\begin{array}{l}\text { Borg } \\
\text { score }\end{array}$ & $\begin{array}{l}\text { Peak } \\
\text { heart } \\
\text { rate } \\
\text { (beats/min) }\end{array}$ & $\begin{array}{l}\text { Exercise } \\
\text { time } \\
\text { (mins) }\end{array}$ & $\begin{array}{l}\text { Borg } \\
\text { score }\end{array}$ & $\begin{array}{l}\text { Peak } \\
\text { heart } \\
\text { rate } \\
\text { (beats/min) }\end{array}$ & $\begin{array}{l}\text { Exercise } \\
\text { time } \\
\text { (mins) }\end{array}$ & $\begin{array}{l}\text { Borg } \\
\text { score }\end{array}$ \\
\hline $\begin{array}{l}\text { AAI } \\
\text { AAIR } \\
\text { p value }\end{array}$ & $\begin{array}{l}122(28) \\
130(22) \\
<0.02\end{array}$ & $\begin{array}{l}12 \cdot 3(4 \cdot 1) \\
12 \cdot 3(3 \cdot 8) \\
\text { NS }\end{array}$ & $\begin{array}{l}17.2(1.4) \\
17.0(1.7) \\
\text { NS }\end{array}$ & $\begin{array}{l}104(17) \\
120(5) \\
<0.003\end{array}$ & $\begin{array}{l}11.4(4.4) \\
11.4(4.0) \\
\text { NS }\end{array}$ & $\begin{array}{l}16.8(1 \cdot 2) \\
16.5(1.6) \\
\text { NS }\end{array}$ & $\begin{array}{r}97(13) \\
116(19) \\
<0.002\end{array}$ & $\begin{array}{l}11 \cdot 1(4 \cdot 8) \\
11 \cdot 1(4 \cdot 4) \\
\text { NS }\end{array}$ & $\begin{array}{l}17 \cdot 2(1 \cdot 1) \\
16 \cdot 3(1.7) \\
\text { NS }\end{array}$ \\
\hline
\end{tabular}

treadmill exercise testing by one patient. In the remaining 26 patients the last two exercise tests were compared to reduce the effect of training. Table 1 shows the results for peak heart rate, duration of exercise, and Borg score at peak exercise in AAI $v$ AAIR mode for the group as a whole and for the subgroups with chronotropic incompetence defined by the two methods.

When chronotropic incompetence was defined as peak heart rate $<0.8(220$ - age of the patient in years) the 15 patients included nine patients with activity sensing and six with minute volume sensing systems. Chronotropically incompetent patients with activity sensing systems showed a mean rise in heart rate of 13 (17) beats/min (baseline 100 (19) beats/min, peak exercise 113 (17) beats/min, $\mathrm{p}<0.05$ ), $v 21$ (14) beats/min (NS) in those with minute ventilation sensing systems (baseline 109(13) beats/min, peak exercise 130 (17) beats/min, $p<0.01$ ). Neither system significantly improved the duration of exercise (activity sensing: AAI $10 \cdot 3(5 \cdot 3) \mathrm{min}$, AAIR $10 \cdot 0(4 \cdot 6) \mathrm{min}(\mathrm{NS})$; minute ventilation sensing: AAI $13 \cdot 1(2 \cdot 0)$ min, AAIR 13.4 (1.4) min (NS)).

In 10 of the patients the PR interval could be measured clearly in the AAI and the AAIR exercise tests, at rest, and at peak exercise. In these patients, the PR interval at rest was 152 (27) $\mathrm{ms}$ in the AAI and 154 (31) $\mathrm{ms}$ in the AAIR mode and at peak exercise it was 140 (21) $\mathrm{ms}$ in the AAI and 146 (28) in the AAIR mode. There were no significant differences between any pairs of these values. No patients showed an increase in the PR interval of $>20$ $\mathrm{ms}$ in response to exercise and none showed evidence of second degree AV block in response to exercise.

\section{ARRHYTHMIAS}

During the study two patients progressed to permanent artrial flutter or fibrillation, one when in AAI and one in AAIR mode. One further patient had continuous atrial fibrilla- tion on one out of two 24 hour tapes when in AAI mode, but reverted spontaneously to sinus rhythm when still in AAI mode. These arrhythmias were excluded from the calculations of frequency of supraventricular arrthythmia. Table 2 shows the results for the frequency of atrial and ventricular premature beats, couplets, and runs in both modes for the whole group and for the two subgroups with chronotropic incompetence. No significant differences between the two modes were found in any of the groups.

\section{Discussion}

Adaptive rate pacing has been shown to improve capacity to exercise in ventricular and dual chamber pacing. ${ }^{19-21}$ By extrapolation from these situations, it has been proposed that AAIR pacing is the optimal mode for patients with sick sinus syndrome. ${ }^{45}$ It is predictable that patients with a normal rise in heart rate on exercise would fail to show improvement in duration of exercise with AAIR pacing. We had anticipated, however, that duration of exercise would be improved in patients with chronotropic incompetence who achieved a significantly higher peak heart rate on exercise in AAIR mode.

Problems exist in defining chronotropic incompetence as there is no uniformly accepted method of assessment. We therefore chose to analyse the results by the two most widely used definitions, but found no difference in tolerance of exercise or suppression of atrial arrhythmia, whichever definition was used. The difference in mean peak heart rate between exercise in AAI and AAIR modes in the chronotropically incompetent patients was modest, amounting to only 16 and 19 beats per minute in the two subgroups.

This however reflects programming that would be considered optimal by many centres, as it avoids a rise to an over-rapid heart rate or to high ceiling rates on minimal exertion, which many patients find disconcerting.

Table 2 Arrhythmia data for whole group and patients with chronotropic incompetence (CI) with peak heart rates in $A A I<80 \%$, and $<75 \%$ of age predicted maximum value (all NS)

\begin{tabular}{|c|c|c|c|c|c|c|c|}
\hline & \multirow[b]{2}{*}{ Mode } & \multicolumn{3}{|c|}{ Atrial arrhythmias/h } & \multicolumn{3}{|c|}{ Ventricular arrhythmias/h } \\
\hline & & Isolated & Couplets & Runs & Isolated & Couplets & Runs \\
\hline \multirow{3}{*}{$\begin{array}{l}\text { Whole group } \\
\text { CI } 0.8(220 \text { - age })\end{array}$} & $\begin{array}{l}\text { AAI } \\
\text { AAIR }\end{array}$ & $\begin{array}{l}40(143) \\
52(166)\end{array}$ & $10(44)$ & $\begin{array}{l}6(31) \\
5(24)\end{array}$ & $\begin{array}{r}13(25) \\
9(17)\end{array}$ & $\begin{array}{l}0.3(0.9) \\
0.2(0.4)\end{array}$ & $\begin{array}{l}0.04(0.2) \\
0.02(0.05)\end{array}$ \\
\hline & AAI & $16(33)$ & $0.6(1.3)$ & $0.09(0.12)$ & $14(22)$ & $0.6(1.3)$ & $0.08(0.2)$ \\
\hline & AAIR & $32(75)$ & $2 \cdot 1(6 \cdot 6)$ & $0.57(1.2)$ & $11(22)$ & $0.1(0.3)$ & $0.02(0.04)$ \\
\hline \multirow{2}{*}{ CI $0.75(220$ - age $)$} & AAI & $8(11)$ & $0.3(0.4)$ & $0.09(0.13)$ & $16(25)$ & $0.6(1.4)$ & $0.08(0.2)$ \\
\hline & AAIR & $17(46)$ & $0.4(0.7)$ & $0.12(0.21)$ & $14(25)$ & $0.1(0.3)$ & $0.02(0.04)$ \\
\hline
\end{tabular}


Programming resulted from either following the manufacturer's directions for achieving optimal rate response, or from using settings at the upper end of the range. In the chronotropically incompetent patients the activity sensing mode gave a limited increase above the peak sinus rate (mean peak heart rate difference between AAI and AAIR was 13 (17) beats/min) despite a rate response setting at the time of the AAIR exercise test of 8 (1) (the range of available settings is 1-10). The rate response setting at the time of exercise in the AAIR mode in the chronotropically incompetent group with minute volume sensing systems was 20 (2) (determined through exercise telemetry as described in the programming manual). It is possible that programming to still higher rate response settings would have resulted in a greater difference between peak heart rates in AAI $v$ AAIR mode and might have resulted in a measurable difference in exercise time between the two modes; however, there was no convincing trend in this direction with the small, but significantly higher heart rates found in the AAIR mode.

It is also possible that a small improvement in capacity to exercise in response to adaptive rate pacing was masked by the stepwise increases in cardiovascular and musculoskeletal demand imposed by the exercise protocol. If at some point during the protocol there was a sudden noticeable increase in treadmill slope or speed, many patients might stop at this point and their exercise durations would therefore cluster at this stage. This also seems an unlikely explanation as the CAEP protocol has been designed to produce a smooth increase in metabolic equivalents of the task throughout the exercise (fig) and similar exercise protocols with stepwise increases used in comparison between ventricular fixed and adaptive (VVI and VVIR) pacing, have not prevented the detection of differences in duration of exercise.

Ventricular rate adaptive pacing has been shown to result in improved capacity to exercise in groups of patients with AV block secondary to various underlying diseases ${ }^{1920}$ and in groups including patients with sick sinus syndrome and AV block. ${ }^{2122}$ In these studies patients achieved higher peak heart rates when exercising in VVIR than in VVI mode, and the authors concluded that the improvement in exercise tolerance resulted from the achievement of higher peak cardiac outputs. Measurements of peak cardiac output, however, have failed to show a significant improvement with VVIR compared with VVI pacing. Beyersdorf and colleagues found only a modest increase in cardiac output at peak exercise from $10.6(0.8) \mathrm{l} / \mathrm{min}$ in VVI to 12.7 $(1.5) \mathrm{l} / \mathrm{min}$ in VVIR mode that failed to reach significance. $^{22}$ The difference in peak heart rate between the two groups was 21 beats/min.

In patients with sick sinus syndrome, atrial pacing has haemodynamic advantages when compared with ventricular pacing at rest. ${ }^{23}$ Cardiac output at rest is increased by $20 \%-30 \%$ and there are improvements in left ventricular filling pressure. ${ }^{24}$ The relative importance of heart rate in the determination of cardiac output during exercise in the two modes is uncertain. It is believed that patients with good left ventricular function are less dependent on a rise in heart rate during exercise than those with myocardial damage; ${ }^{25}$ others have shown a limited effect of acceleration of heart rate on cardiac output irrespective of ejection fraction. ${ }^{26}$ Groups of patients with sick sinus syndrome, such as those included in this study, may have better left ventricular function than patients with mixed aetiologies that give rise to AV block. Cardiac output in response to exercise may therefore be less dependent on rate in patients with sick sinus syndrome than in patients with AV block. The abnormal ventricular activation pattern resulting from ventricular pacing may further limit the heart's ability to modulate stroke volume in response to exercise, and may make patients with ventricular pacing more dependent on acceleration of heart rate.

Cardiac output may not be the only factor affecting capacity to exercise in patients with sick sinus syndrome. Other physiological mechanisms that make an important contribution to the response to exercise are: redistribution of blood flow away from non-exercising regions such as the kidneys and splanchnic bed and towards exercising skeletal muscle, the capacity of the blood to carry and dissociate oxygen, the ability of exercising muscle to extract oxygen, the ability to clear and to tolerate increasing blood lactate concentrations, the compliance of the lungs and diaphragmatic strength, pulmonary gas exchange, skeletal muscle strength, and neuromuscular control. If one of these mechanisms were the limiting factor in determining tolerance to exercise, heart rate would not influence the maximal capacity to exercise. The contribution of heart rate to cardiac output only becomes predominant over stroke volume at higher levels of exertion. ${ }^{27}$ At the point where exercise is limited by the integrated response of the cardiovascular system, the rise in cardiac output may still be principally dependent on stroke volume.

One potential limitation of AAIR pacing on exercise that has been explored is the development of second degree heart block due to rapid acceleration of the atrial paced rate. This results in the occurrence of atrial activity before AV nodal recovery. ${ }^{1328}$ Despite the steep rate response slopes programmed during the AAIR phases of this study, we did not find this. In the patients in whom the response of the PR interval to exercise could be measured with confidence, there seemed to be little shortening of the PR interval at peak exercise. This was a feature of both the sinus (AAI) and atrially paced (AAIR) rhythms during exercise. It is possible that a failure of normal shortening of the AV interval may impair optimal ventricular filling at peak exercise and may have limited the benefits gained from increased heart rate in the AAIR patients.

As well as assessing the effect of AAIR pacing on capacity to exercise, we studied the efficacy of AAIR pacing in suppressing arrhythmias. Both AAIR and dual chamber 
adaptive rate (DDIR) pacing have been proposed for the tachy-brady form of sick sinus syndrome. ${ }^{9}$ Preliminary data have suggested a lower incidence of atrial arrhythmias with the AAIR mode in patients with sick sinus syndrome. ${ }^{14}$ These results were from a non-randomised parallel group study, however, with only seven patients in the AAIR group and the authors suggested that a further study with larger numbers was required. In our study, we found no evidence of overall proarrhythmic or antiarrhythmic effects in the group of patients as a whole, or in the patients with chronotropic incompetence irrespective of the definition of chronotropic incompetence used. It is possible that beneficial antiarrhythmic effects of AAIR may exist, but they are balanced by proarrhythmic influences. Sensor driven atrial pacing may occur within the vulnerable period of atrial repolarisation and thus there is a risk of inducing atrial arrhythmias. This situation may also exist in dual chamber pacing. The DDDR mode was at first thought to inhibit atrial extrasystoles and to lower the incidence of atrial arrhythmias; however, one study has suggested that the long-term use of DDDR pacemakers is associated with a higher incidence of atrial arrhythmias compared with conventional DDD pacing ${ }^{29}$ and no antiarrhythmic effect was found in a crossover study between DDD and DDDR modes in a mixed group of patients with sick sinus syndrome and AV block. ${ }^{30}$

In conclusion, this study failed to show benefits in either maximal capacity to exercise or suppression of arrhythmia from AAIR pacing compared with fixed rate AAI pacing, even in patients with chronotropic incompetence of the sinus node. The use of AAIR pacing entails greater expense and increased time and operator skill to achieve appropriate programming of the rate response variables. The British Pacing and Electrophysiology Group Working Party has gone so far as to recommend the use of AAIR or DDDR as the optimal modes in sick sinus syndrome in general. There has, however, been no convincing evidence for the supposed benefits of these modes in otherwise healthy patients with sick sinus syndrome. We see little indication for the implantation of AAIR systems in such patients. Current recommendations for the use of this pacing mode should probably be revised.

We are grateful to Jan Poloniecki $\mathrm{PhD}$ for advice with the statistical analysis of the results. G A Haywood was supported by a British Heart Foundation Junior Research Fellowship.

1 Ryden L. Atrial inhibited pacing - an underused mode of cardiac stimulation. PACE 1988;11:1375-9.

2 Rosenquist M. Atrial pacing for sick sinus syndrome. Clin Cardiol 1990;13:43-7.

3 Kolettis T, Miller H, Boon N. Atrial pacing: who do we pace and what do we expect? Experience with 100 atrial pacemakers. PACE 1990;13:625-30.

4. Dreifus L, Fisch C, Griffin J, Gillette P, Mason J, Parsonnet V. ACCAAHA task force report: guidelines forsonnet V. ACCAAHA task force report: guidelines for implantation of cardiac pacemakers and a

5 Clarke $M$, Sutton R, Ward D, et al. Recommendations for Clarke $\mathrm{M}$, Sutton $\mathrm{R}$, Ward for symptomatic bradycardia. $\mathrm{Br}$ Heart f 1991;66:185-91.

6 Sutton R, Kenny R-A. The natural history of sick sinus syndrome. $P A C E$ 1986;9:110-4.

7 Rosenquist $\mathrm{M}$, Obel I. Atrial pacing and the risk for AV block: is there a time for change in attitude? $P A C E$ 1989;12:97-101

8 Haywood G, Ward J, Ward D, Camm AJ. Atrioventricular Wenckebach point and progression to AV block in sinoatrial disease. PACE 1990;13:2054-8.

9 Sutton R. DDDR pacing PACE 1990;13:385-6.

10 Simonsen E. Assessment of the need for rate responsive pacing in patients with sinus node dysfunction: a prospective study of heart rate response during daily activities and exercise testing [abstract]. $P A C E$ 1987; 10:418.

11 Prior M, Masterson M, Morant V, Castle L, Maloney J. Do patients with sinus node dysfunction and permanent pacemakers require an additional chronotropic sensor? [abstract.] PACE 1987;10:418.

12 Hatano $K$, Kato $R$, Hayashi $H$, Noda $S$, Sotobata I, Murase $M$. Usefulness of rate responsive atrial pacing in parients with sick sinus syndrome. $P A C E$ 1989;12: 16-24.

13 Pouillot C, Daubert C, Mabo P, Cazeau S, Paillard F, Le Breton $\mathrm{H}$. The lack of adaptation in PR interval to heart rate: a frequent limitation for AAIR pacing [abstract]. PACE 1990;13:504.

14 Kato R, Terasawa T, Gotoh T, Suzuki M. Antiarrhythmic efficacy of atrial demand (AAI) and rate responsive atrial pacing. In: Santini $M$, Pistolese $M$, Alliegro A, ed. Progress in clinical pacing. Amsterdam: Excerpta Medica 1988:15-24.

15 Frye R, Collins J, DeSanctis $\mathrm{R}$, et al. Guidelines for permanent pacemaker implantation, May 1984. Joint ACC/AHA task force report. $₹ \mathrm{Am}$ Coll Cardiol 1984 4:434-42.

16 Wilkoff B, Corey J, Blackburn G. A mathematical model of the chronotropic response to exercise. $\mathcal{F}$ Electrophysiol 1989;3:176-80.

17 Gwinn N, Leman R, Zile M, Kratz J, Gillette P. Pacemaker patients become chronotropic incompetent with time [abstract]. PACE 1990;13:535.

18 Isaeff $D$, Jutzy R, Florio J. Programming of rate responsive pacemakers. F Electrophysiol 1989;3:202-16.

19 Gammage M, Schofield S, Rankin I, Bennet M, Coles P, Pentecost B. Benefit of single setting rate responsive ventricular pacing compared with fixed rate demand pacing in elderly patients. PACE 1991;14:174-80.

20 Hedman A, Norlander R. QT sensing rate responsive pacing compared to fixed rate ventricular inhibited pacing: a controlled clinical study. $P A C E$ 1989;12:374-85

21 Oto $M$. Müderrisoglu H, Ozin M, et al. Quality of life in patients with rate responsive pacemakers: a randomised, patients with rate responsive pacemakers

22 Beyersdorf F, Kreuzer J, Happ J, Zegelman M, Satter P. Increase in cardiac output with rate-responsive pacemaker. Ann Thorac Surg 1986;42:201-5.

23 Wirtzfeld A, Schmidt F, Himmler F, Stangl K. Physiological pacing: present status and future developments. PACE 1987;10:41-57.

24 Sutton R, Citron P. Electrophysiological and haemodynamic basis for application of new pacemaker technology in sick sinus syndrome and atrioventricular block. Br Heart f 1979;41:600-12.

25 Sowton E. Haemodynamic studies in patients with artificial pacemakers. Br Heart f 1964;26:737-46.

26 Buckingham $T$, Woodruff $\mathrm{R}$, Pennington $\mathrm{D}$, et al. Effect of ventricular function on the exercise haemodynamics of variable rate pacing. $₹ \mathrm{Am}$ Coll Cardiol 1988;11: of variable

27 Jones N. Clinical exercise testing. Philadelphia: W B Saunders, 1988:44-6.

28 Den Dulk K, et al. Pacemaker syndrome with AAI rate variable pacing: importance of atrioventricular conduction properties, medication and pacemaker programmability. PACE 1988;11:1226-31

29 Feuer J, Shandling A, Ellestad M. Senor modulated dual chamber cardiac pacing: too much of a good thing too fast? PACE 1990;13:816-8.

30 Spencer W, Markowitz T, Alagona P. Rate augmentation and atrial arrhythmias in DDDR pacing. $P A C E$ 1990;13:1847-51.

Appendix 1 Chronotropic assessment exercise protocol

\begin{tabular}{|c|c|c|c|c|}
\hline Stage & $\begin{array}{l}\text { Speed } \\
(m p h)\end{array}$ & $\begin{array}{l}\text { Grade } \\
(\%)\end{array}$ & $\begin{array}{l}\text { Time } \\
(\min )\end{array}$ & Mets \\
\hline $\begin{array}{l}\text { Warm up } \\
1 \\
2 \\
3 \\
4 \\
5 \\
6 \\
7 \\
8 \\
9 \\
10 \\
11\end{array}$ & $\begin{array}{l}1 \cdot 0 \\
1 \cdot 0 \\
1.5 \\
2 \cdot 0 \\
2 \cdot 5 \\
3 \cdot 0 \\
3 \cdot 5 \\
4 \cdot 0 \\
5 \cdot 0 \\
6 \cdot 0 \\
7 \cdot 0 \\
7 \cdot 0\end{array}$ & $\begin{array}{r}0 \\
2 \\
3 \\
4 \\
5 \\
6 \\
8 \\
10 \\
10 \\
10 \\
10 \\
15\end{array}$ & $\begin{array}{l}2 \\
2 \\
2 \\
2 \\
2 \\
2 \\
2 \\
2 \\
2 \\
2 \\
2 \\
2 \\
2\end{array}$ & $\begin{array}{r}1 \cdot 5 \\
2 \cdot 0 \\
2 \cdot 8 \\
3 \cdot 6 \\
4 \cdot 6 \\
5 \cdot 8 \\
7 \cdot 5 \\
9 \cdot 6 \\
12 \cdot 1 \\
14 \cdot 3 \\
16 \cdot 5 \\
19 \cdot 0\end{array}$ \\
\hline
\end{tabular}

Appendix 2 Borg scale: scoring of perceived exertion

\begin{tabular}{ll}
\hline Symptoms & Borg scale \\
\hline Very very light & $6-7$ \\
Very light & $8-9$ \\
Fairly light & $11-12$ \\
Somewhat hard & $13-14$ \\
Hard & $14-15$ \\
Very hard & $16-17$ \\
Very very hard & $18-20$ \\
\hline
\end{tabular}

